\title{
ANÁLISIS DEL PATRÓN DE COMPORTAMIENTO DEL CONSUMIDOR DE QUITO, ECUADOR
}

\section{PATTERN ANALYSIS OF CONSUMERS' BEHAVIOR IN QUITO, ECUADOR}

Guido Marcelo Salazar Lozada*, José Félix García Rodríguez**, Luis Arturo Méndez Olán***

*Maestro en Ciencias. Facultad de Ciencias Administrativas de la Universidad Tecnológica Equinoccial (UTE), Ecuador. Email: guido.salazar@ute.edu.ec. ORCID: https://orcid.org/0000-0003-3673-1326.

**Doctor en Finanzas Públicas. División Académica de Ciencias Económico Administrativas de la Universidad Juárez Autónoma de Tabasco. ORCID: https://orcid.org/0000-0002-7319-1472.

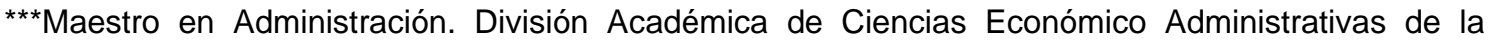
Universidad Juárez Autónoma de Tabasco. Email: luis_arturo79@hotmail.com.mx. ORCID: https://orcid.org/0000-0001-7025-8777.

Dirección para recibir correspondencia: jfgr55@hotmail.com.mx 
ANÁLISIS DEL PATRÓN DE COMPORTAMIENTO DEL CONSUMIDOR DE QUITO, ECUADOR.

\section{RESUMEN}

Este estudio presenta las preferencias -según su prioridad- de compra de bienes principales y comunes para todas las personas que habitan en la ciudad en un momento específico y en una situación económica del país propia de la época -año 2018-.

OBJETIVO: Proporcionar información del comportamiento del consumidor de Quito, cuando se trata de adquirir bienes necesarios y que por diferentes factores no los pueden obtener.

MATERIAL Y MÉTODO: Investigación con enfoque mixto, en la cual se aplicaron dos métodos de investigación. Para la parte cualitativa, a través de un grupo focal, se utilizó el método inductivo, así como la elaboración de preguntas de la encuesta aplicada, y con el apoyo del método deductivo se realizó la investigación cuantitativa, aplicada a 626 consumidores.

RESULTADOS: Las prioridades del consumidor de Quito, son los alimentos, los cuales compran en supermercados; los electrodomésticos los adquieren en tiendas especializadas, buscando almacenes con variedad de marcas, toman la decisión de compra sin consultar y pagan de contado bienes menores a $\$ 500$.

CONCLUSIONES: Los alimentos son su primera prioridad, por lo que se entiende que en épocas de recesión económica se privará de muchas adquisiciones que no sean alimenticias. Asimismo, los proveedores de alimentos deben centrarse en tener mayor presencia en los supermercados.

PALABRAS CLAVE: Teoría del consumidor. Comportamiento de compra. Prioridades de consumo. Bienes principales.

\section{ABSTRACT}

This study shows purchases preferences -according to priority- of commun and main goods for all the people that inhabit there in a specific period of time and in a specific economic situation of the country -year 2018-. 
ANÁLISIS DEL PATRÓN DE COMPORTAMIENTO DEL CONSUMIDOR DE QUITO, ECUADOR.

OBJECTIVE: To provide information of consumer behavio in Quito, when adquiring necessary goods and for different reasons they fail to succeed.

MATERIAL AND METHOD: A mixed research, for the qualitative section, a focus group was implemented with an inductive method; and for the quantitative section, a survey was administered to 626 consumers with a deductive method.

RESULTS: Consumers' priority in Quito is food, which is acquired in supermarkets; appliances are purchased in specialized stores, they make their purchase decision without consulting and pay in cash for goods that are worth less than $\$ 500$.

CONCLUSIONS: Being food their priority, it is inferred that in times of economic recession, they avoid buying non-nourishing goods. Food suppliers must concentrate then in having more presence in supermarkets.

KEY WORDS: Consumer theory. Purchasing behavior. Consumer prorities. Main goods.

\section{INTRODUCCIÓN}

El comportamiento del consumidor es un tema que tiene principio pero no tiene fin. Inició con la preocupación de grandes investigadores, profesionales en diferentes disciplinas que propusieron en su época distintas teorías y modelos de comportamiento. Con el avance tecnológico y científico, los estudios del comportamiento de las personas ante un impulso se ha facilitado sustancialmente, debido a que en la actualidad se utilizan herramientas de laboratorio de Neurociencia y Neuromarketing para interpretar con mayor certeza la respuesta del consumidor ante dicho estímulo. Los equipos tecnológicos destinados para las pruebas y análisis del comportamiento del consumidor, pueden leer las ondas cerebrales directamente, y luego a través de software especial, ser interpretadas y puestas a consideración de los especilistas del marketing y comunicación, para la elaboración de las estrategias de comercialización.

Como se mencionó anteriormente, que el comportamiento del ser humano ha sido abordado desde distintas disciplinas, el psicoanalista Sigmund Freud, citado por Schiffman, L. \& Lazar Kanuk, L. (2010), en su teoría relaciona las necesidades y los impulsos inconscientes como parte de la personalidad humana y de su motivación a la toma de decisiones. Para fundamentar su teoría, Freud realizó un estudio que evaluaba las experiencias vividas en la SALAZAR-LOZADA G. M., FÉLIX-RODRíGUEZ J. F., MÉNDEZ-OLÁN L. A. 
ANÁLISIS DEL PATRÓN DE COMPORTAMIENTO DEL CONSUMIDOR DE QUITO, ECUADOR.

niñez temprana del individuo, en el análisis de los sueños, y los problemas de adaptación mental y física de sus pacientes, a partir de este análisis, se definió que la personalidad humana consta de tres sistemas relacionados: el "ello", el "súper yo" y el "yo.

Según la teoría freudiana, el "ello" es la parte irreflexiva y primitiva del ser humano, que lo orienta a satisfacer sus necesidades físicas con la mayor inmediatez posible, sin pasar por la reflexión previamente. Este sistema se enfoca en la satisfacción del hambre, la sed, el deseo sexual, entre otras necesidades básicas del ser humano.

Mientras que el "súper yo" es lo contrario del "ello"; este sistema es la parte reflexiva que actúa en base a la conducta moral y ética de la sociedad a la que pertenece el individuo, mismo que inhibe las fuerzas impulsivas del "ello". Por lo tanto, dependerá de la moral y la ética con la que fue educado el individuo para actuar y satisfacer sus necesidades, dejando de lado el impulso inconsciente

En su libro "La teoría de la clase ociosa", Thorstein Veblen, citado por Fernández Delgado (2014), describe las características del comportamiento de personas con poder adquisitivo, Veblen, Psicólogo Social, pretende estudiar el lugar y el valor de la clase ociosa como factor económico de la vida moderna: la emulación, el ocio, el consumo ostensible, los gustos y la moda entre otros elementos, constituyen variables indispensables que hay que conocer desde el punto de vista de una teoría económica, y no circunscribirse únicamente a la racionalidad y la transitividad de las preferencias. Veblen menciona el consumo afamado, que es el gasto de dinero para adquirir bienes de lujo, para mostrarlos públicamente y que los demás vean su poder económico, mostrando un status social y una superioridad.

El economista John Maynard Keynes citado por Lavoie (2005) propone la Teoría General del Empleo, el interés y el dinero, en una época llamada la Gran Depresión económica mundial en la década de los años 30. Keynes analizó la propensión marginal al consumo y su efecto en la economía; es decir, si una persona incrementa su renta, cuánto incrementa su consumo y dedujo que un individuo con renta alta destinará a su consumo una parte de su incremento y la diferencia la ahorrará, mientras que una persona de renta baja destinará todo su incremento al consumo.

La teoría del consumidor postkeynesiana se alimenta de numerosas fuentes, en especial de los trabajos de psicólogos, socio economistas, institucionalistas, especialistas en mercadotecnia, y de ciertos economistas como Nicholas GeorgescuRoegen o Herbert Simon y su escuela behaviorista. Esta teoría no se apoya en un 
ANÁLISIS DEL PATRÓN DE COMPORTAMIENTO DEL CONSUMIDOR DE QUITO, ECUADOR.

enfoque axiomático; sino en unos principios que dan razón de los comportamientos observados (Lavoie, 2005, p. 36).

a) Racionalidad procedimental (Simon): "El consumidor se regiría por hábitos, por reglas de comportamiento no compensadoras, por atajos" (Lavoie, 2005, p. 36).

b) Saciedad (Georgescu-Roegen): "Más allá de un umbral finito, la necesidad queda satisfecha, y consumir más unidades no aumenta la satisfacción" (Lavoie, 2005, p. 36).

c) Separación (Lancaster): "El consumidor subdivide sus elecciones o sus necesidades en diversas categorías, débilmente relacionadas unas con otras" (Lavoie, 2005, p. 36).

d) Subordinación (Georgescu-Roegen): "Las necesidades a menudo están jerarquizadas y subordinadas unas a otras" (Lavoie, 2005, p. 36).

e) Crecimiento (Georgescu-Roegen, Pasinetti): "El tiempo y el aumento de ingresos permiten pasar de una necesidad a otra de forma escalonada" (Lavoie, 2005, p. 36).

f) Dependencia (Galbraith): "Las necesidades están influidas por la publicidad, las modas, los grandes personajes, la cultura, la familia, los amigos" (Lavoie, 2005, p. 36).

g) Herencia (Georgescu-Roegen): "Las elecciones de hoy están condicionadas por las elecciones de ayer" (Lavoie, 2005, p. 36).

El comportamiento de compra del consumidor se basa en un orden de necesidades que satisfacer. Abraham Maslow citado por López A. (2013), presenta en su teoría diferentes niveles de necesidades del ser humano representadas jerárquicamente en una pirámide. En la base ubica las necesidades básicas y las necesidades superiores, mientras van ascendiendo hacia la cumbre de la pirámide.

El pasar de un nivel a otro depende del grado de satisfacción que va obteniendo en cada nivel, de tal manera que, si satisface las necesidades básicas, como la alimentación, vestimenta, abrigo, podrá ir cubriendo las superiores como seguridad, afiliación, reconocimiento, autorealización.

Los especialistas, Herrera, Ramírez, Roa y Herrera I. (2004) afirman que la motivación es uno de los elementos fundamentales para explicar la conducta del ser humano relacionada con el porqué de su comportamiento. Dicho de otra manera, es el impulso para que una persona inicie una acción (activación), trace un objetivo (dirección) e intente persistentemente alcanzarlo (mantenimiento).

Schiffman, L. \& Lazar Kanuk, L. (2010), exponen que en la Teoría del Aprendizaje se considera a todos los organismos como neutrales, a quienes se puede enseñar conductas a través de la repetición. El aprendizaje por estímulo-respuesta se basa en la premisa de que, 
ANÁLISIS DEL PATRÓN DE COMPORTAMIENTO DEL CONSUMIDOR DE QUITO, ECUADOR. respuestas observables ante estímulos externos específicos indican que ocurrió un aprendizaje. Cuando una persona actúa (responde) de manera predecible ante un estímulo conocido, se dice que "ha aprendido".

Es fundamental destacar que existen varios modelos y teorías del comportamiento del consumidor, que no se les ha descrito en este documento y que tienen su relevancia y aplicación según el tema a investigar, los cuales son: Modelo de Marshall desde la perspectiva económica, Freud desde lo psicoanalítico, Nicosia basado en la relación informativa afectiva, Howard-Seth selección de Marca, Schiffman desde el punto de vista de la toma de decisiones y O'Shaughnessy se ha centrado en la compra no planificada.

Esta investigación, tiene como resultado la selección de productos y servicios, por parte del consumidor, según la importancia y preferencia que éstos tienen para cubrir las necesidades prioritarias

Una vez concluída la busqueda de información documental y datos estadísticos relacionados al comportamiento del consumidor de Quito-Ecuador, se concluye que existe poca información respecto al tema.

Las investigaciones especificas con un direccionamiento en particular, es común encontrarlas en los estudios realizados hasta el momento. Estos estudios han servido para suplir un vacío existente de información muy particular, necesaria y requerida como soporte en la toma de decisiones empresariales, es posible contar con información del comportamiento del consumidor ecuatoriano de una industria específica, como alimentos, educación, puntos de venta, almacenes, centros comerciales y otros.

En este estudio, la recolección de datos del comportamiento del consumidor de Quito, se basó en las metodologías tradicionales empleadas en investigación de mercados; es decir, grupo focal -focus group- para la investigación cualitativa y una encuesta física para la obtención de datos porcentuales en el estudio cuantitativo.

La investigación detalla el comportamiento general de compra de los habitanes de la ciudad de Quito en el momento de decidir por un bien o servicio, considera para tal efecto, sus prioridades económicas, de satisfacción personal o de busqueda de reconocimiento.

El estudio valora los hábitos generales del gasto de las personas en un tiempo determinado año 2018- y bajo el nuevo ambiente económico de la época. Los hábitos generales se los obtuvo del grupo focal -focus group- con el que se inició la investigación, compuesto por trece personas, cuyo detalle se lo describe en el siguiente acápite - método de la investigación-. 
ANÁLISIS DEL PATRÓN DE COMPORTAMIENTO DEL CONSUMIDOR DE QUITO, ECUADOR.

Los temas seleccionados por el grupo, bajo consenso, para iniciar la elaboración del instrumento -encuesta- fueron los siguientes:

1. Prioridad de los gastos del año.

2. Lugar de preferencia de compra de comestibles.

3. Lugar de preferencia de compra de electrodomésticos.

4. Lugar de preferencia de compra de ropa.

5. Lo que le gusta más del lugar de compra.

6. Lo prioritario en el lugar de compra respecto al producto.

7. La influencia externa en la decisión de compra.

8. La forma de pago que prefiere para sus compras hasta $\$ 500$.

9. Énfasis en las características generales de cualquier producto.

10. Razones para la compra de un producto.

11. Razones para aprovechar las oportunidades de compra.

12. Influencia de los anuncios publicitarios en la decisión de compra.

Los resultados de la investigación son un aporte adicional a la información del consumidor de la ciudad y está a la disposición de quienes quieran enriquecerla, en bien de la empresa y la Academia.

\section{MATERIAL Y MÉTODO}

El enfoque de esta investigación, mencionado anteriormente, requirió de dos métodos de investigación que se aplicó directamente; para la parte cualitativa, a través de un grupo focal, se utilizó el método inductivo y con el resultado de este ejercicio grupal se elaboró las preguntas de la encuesta y con el apoyo del método deductivo, se realizó la investigación cuantitativa a 626 personas.

El estudio inició con la investigación exploratoria formal (Roberto Hernández, Carlos fernández, Pilar Baptista, 2014). Se reunió a 13 consumidores quiteños, con capacidad económica media y media alta, género masculino y femenino, edades entre 18 y 54 años, con hábitos de consumo normales o en su lugar, personas encargadas de las compras de la familia. De los resultados de esta reunión se obtuvo información muy relevante para la construcción del instrumento. 
ANÁLISIS DEL PATRÓN DE COMPORTAMIENTO DEL CONSUMIDOR DE QUITO, ECUADOR.

De los temas propuestos por el grupo de personas participantes, se elaboró las preguntas de preferencia o prioridad de compra. En cada pregunta, el encuestado debía seleccionar el orden de preferencia entre cinco alternativas planteadas por pregunta.

El objetivo que se buscó con este tipo de preguntas, fue lograr que cada participante de la encuesta enumere el orden de prioridad de compra de las alternativas presentadas, de esta manera se pudo contabilizar cuántas personas seleccionaron la primera alternativa como prioridad uno, cuántas como prioridad dos, cuántas como la tercera prioridad y así sucesivamente.

En definitiva, una vez procesados los datos, se decidió sumar los valores correspondientes a las respuestas de prioridad uno y prioridad dos de cada alternativa por ser los valores más representativos, según criterio y opinión de los encuestados. La metodología utilizada para esta parte facilitó la interpretación de los resultados y el orden de preferencia de las opciones.

Los resultados de las alternativas y su correspondiente prioridad, se los registró en una tabla muy sencilla y de facil interpretación (ver tabla 1):

Tabla 1

Ejemplo: prioridad de los gastos al año

\begin{tabular}{cccccc}
\hline Prioridad & Alimentos & Ropa & Electrodomésticos & $\begin{array}{c}\text { Artículos } \\
\text { electrónicos }\end{array}$ & Diversión \\
\hline 1 & 459 & 76 & 40 & 54 & 68 \\
2 & 54 & 284 & 80 & 68 & 101 \\
3 & 32 & 131 & 157 & 114 & 153 \\
4 & 15 & 86 & 145 & 231 & 114 \\
5 & 66 & 49 & 204 & 159 & 190 \\
Muestra & 626 & 626 & 626 & 626 & 626 \\
Suma 1 & 513 & 360 & 120 & 122 & 169 \\
\hline
\end{tabular}

Fuente: Elaboración propia.

Es importante mencionar, que para la obtención de estos resultados se realizó la encuesta física e individual, no se consideró aplicarlo digitalmente, debido a que la información a obtener era de gran trascendencia y en este sentido, el contacto físico ayudaba a resolver inquietudes del encuestado y garantizaba una respuesta lo más apegada a la verdad. 
ANÁLISIS DEL PATRÓN DE COMPORTAMIENTO DEL CONSUMIDOR DE QUITO, ECUADOR.

En vista de la importancia de los datos a recopilar, se capacitó profundamente al personal que realizó la encuesta, quedaron en condiciones de resolver inquietudes de toda índole respecto a las preguntas descritas en el instrumento. En resumen, se buscaba obtener la mayor confiabilidad posible en todos los datos obtenidos.

Según el INEC (2017) - Instituto Nacional de Estadística y Censos- el 57.7\% de la población de Quito tiene empleo; por tanto, de los 1.911 .966 habitantes que tiene la ciudad, últimos datos de Ecuadorcifras (2017), la población economicamente activa de la ciudad en el 2017 fue de 1.103.204 habitantes, cifra utilizada para el cálculo de la muestra.

Con una población que sobrepasa el millón de personas -infinita- y una variable cualitativa comportamiento- a investigar, se aplicó la formula correspondiente para obtener el número de habitantes a ser encuestados. Entre las características seleccionadas con las que debían contar las personas que colaborarían con la encuesta, se destacaron, poder adquisitivo moderado (ingresos mensuales mayores a 500 dólares), hábito de compra y poder de decisión.

El tipo de muestreo seleccionado para esta investigación fue no probabilístico por juicio. Los datos que se utilizaron para la aplicación de la fórmula del cálculo de la muestra San Martín (2008), se los detalla a continuación.

$$
\begin{aligned}
& N=1.103 .204 \text { habitantes } \\
& n=\text { muestra } \\
& E=5 \% \\
& p=50 \% \\
& q=50 \% \\
& \qquad n=\left(K^{\star} K{ }^{\star} p * q\right) / E * E \\
& n=(1.96 * 1.96)^{\star} 0.5^{\star} 0.5 / 0.05^{\star} 0.05 \quad n=384
\end{aligned}
$$

La muestra obtenida para esta investigación reveló 384 personas; sin embargo, para conseguir mayor información y más confiabilidad en los resultados, se decidió duplicar la muestra y aplicar el instrumento a 768 habitantes de la ciudad. Una vez realizado el trabajo de campo, se revisaron físicamente todas las las encuestas y se separaron todas aquellas que presentaban algún error en la respuesta a cualquiera de las preguntas, como dejarlas en blanco, no contestar completamente la pregunta, borrones, tachones, contestar con un número fuera del rango permitido para el orden de prioridad o alguna alteración física que 
ANÁLISIS DEL PATRÓN DE COMPORTAMIENTO DEL CONSUMIDOR DE QUITO, ECUADOR.

dejara duda. De esta revisión individual de separación de las encuestas con falla, quedaron para el análisis y obtención de información relevante 626 encuestas.

Para el trabajo de campo, se capacitó a 70 estudiantes de la carrera de Marketing de la universidad UTE-Quito, se zonificó la ciudad y se les entregó 10 encuestas a cada uno, las 68 restantes fueron aplicadas por el investigador directamente a los profesores y administrativos de la Facultad de Ciencias Empresariales.

Para probar el instrumento y la metodología de recopilación utilizada, días antes de lanzar la encuesta final, se realizó un piloto con 25 encuestados, ejercicio que sirvió para afinar texto y redacción.

\section{Diseño de la investigación}

Para este estudio en particular, fue adecuado hacer uso de la investigación no experimental transversal (Roberto Hernández, Carlos fernández, Pilar Baptista, 2014). Las variables consideradas en la investigación del comportamiento del consumidor, se las utilizó en un momento determinado para una situación específica, no se las volverá a requerir por algún tiempo, quizá se las retome para un nuevo análisis, si las condiciones del mercado o las tendencias externas de comportamiento, logren afectar directamente la forma de pensar y actuar del consumidor de Quito-Ecuador. Toda la información obtenida en esta investigación correponde al año 2018, en unas condiciones económicas propias de la época de transición política en Ecuador y un comportamiento de compra propicio para el análisis.

Los datos que corresponden a las variables selecionadas, son recopilados en el momento de la aplicación del instrumento, luego mediante un proceso interno, son cuantificados y analizados utilizando la herramienta Excel. Como resultado del ejercicio, se deriva un diagnóstico y se emiten conclusiones de la investigación. El proceso llevado acabo cae dentro de una investigación con carácter descriptivo y prospectivo (Roberto Hernández, Carlos fernández, Pilar Baptista, 2014).

\section{Resultados de la investigación cuantitativa}

Los datos obtenidos en la encuesta de doce preguntas sobre el comportamiento estándar de compra de los habitantes de la ciudad de Quito, a una muestra de 626 personas, son escenciales para el estudio, se los describe en foma individual en primera insatancia, para luego hacer un compendio. 
Tabla 2

Lista de las preguntas y alternativas para la selección de prioridad

1- Prioridad de los gastos al año.
Alimentos
Ropa
Electrodomésticos
Artículos
electrónicos
Diversión
Ocio

2- Lugar de preferencia de compras de comestibles.

Supermercados Micromercados El mercado Tienda de barrio Central de abastos

3- Lugar de preferencia de compra de electrodomésticos.

Almacenes

especializados

Supermercados WEB-Internet

Venta Informal

En viajes al exterior

4- Lugar de preferencia de compra de ropa.

Almacenes con

variedad de

Especialistas en tipo de ropa

En Supermercados

WEB-Internet

Almacenes de ropa usada

5- Lo que le gusta del lugar de compra.

\author{
Que tenga \\ promociones \\ Orden en la \\ distribución de los
} productos

\author{
Ubicación y \\ estacionamiento
}

Que tenga facilidades de pago

6- Lo prioritario en el lugar de compra respecto al producto.

Que ofrezcan productos de calidad
Productos y maracas preferidas
Productos de última versión/ frescos

7- La influencia externa en la decisión de compra.

Lo decido yo sin consultar a nadie

\section{Consulto con alguien \\ de mi familia para} decidir
Consulto con

amigos o $\mathrm{mi}$ pareja
Consulto en redes sociales

\section{Empleados serviciales y amables}

Productos con precios competitivos $y$ buenas ofertas

8- La forma de pago que prefiere para sus compras hasta $\$ 500$.

Efectivo o cheque Tarjeta de débito $\begin{gathered}\text { Un solo pago con } \\ \text { tarjeta de crédito } \\ \text { sin intereses }\end{gathered} \quad \begin{gathered}\text { Tarjeta de crédito } \\ \text { diferido sin intereses }\end{gathered}$

Crédito directo del lugar de compra

9- Énfasis en las características generales de cualquier producto.

$\begin{array}{lcc}\text { Información } & \text { Compatible con mi } & \text { Práctico y fácil de } \\ \text { detallada de sus } & \text { estilo de vida } & \text { usar } \\ \text { características } & \end{array}$

Que tenga ingredientes ecológicos

Que cumpla con sus promesas

\section{0- Razones por la que compra un producto.}

$\begin{array}{cccc}\begin{array}{c}\text { Para ganar } \\ \text { prestigio ante los } \\ \text { demás }\end{array} & \begin{array}{c}\text { Porque está de moda y } \\ \text { mis amigos lo tienen }\end{array} & \begin{array}{c}\text { Me ayuda a } \\ \text { sentirme mejor al } \\ \text { adquirirlo }\end{array} & \begin{array}{c}\text { Me siento } \\ \text { identificado con los } \\ \text { que compran }\end{array} \\ \begin{array}{c}\text { Compro porque se } \\ \text { agota }\end{array} & \begin{array}{c}\text { El precio es muy } \\ \text { conveniente }\end{array} & \begin{array}{c}\text { Porque la relación } \\ \text { calidad precio es } \\ \text { inmejorable }\end{array} & \begin{array}{c}\text { Por reemplazar un } \\ \text { producto que } \\ \text { cumplió su ciclo de } \\ \text { vida }\end{array}\end{array}$

Me siento más seguro ante los demás

Porque al hacerlo contribuyo a una buena causa

12- Influencia de los anuncios publicitarios en la decisión de compra. 
ANÁLISIS DEL PATRÓN DE COMPORTAMIENTO DEL CONSUMIDOR DE QUITO, ECUADOR.

\begin{tabular}{ccccc}
\hline $\begin{array}{c}\text { Una promoción } \\
\text { visible y }\end{array}$ & $\begin{array}{c}\text { Siempre busco } \\
\text { anuncios de } \\
\text { conveniente me } \\
\text { promociones de mi } \\
\text { incita a la compra }\end{array}$ & $\begin{array}{c}\text { Si no hay } \\
\text { anuncios de } \\
\text { promoción de mi } \\
\text { producto igual lo }\end{array}$ & $\begin{array}{c}\text { No tengo } \\
\text { preferencia de } \\
\text { marca compro el } \\
\text { producto anunciado } \\
\text { en promoción }\end{array}$ & $\begin{array}{c}\text { No importa otras } \\
\text { comprociones yo }\end{array}$ \\
\hline
\end{tabular}

Fuente: Elaboración propia.

La primera pregunta tiene relación directa con los gastos más importantes de las personas que viven en la ciudad de Quito, en el período de un año. Claramente se refleja, en este estudio, que los alimentos con un $\mathbf{8 1 . 9 5 \%}$ es la opción de mayor prioridad, seguida de la vestimenta con $57.51 \%$, la diversión-ocio $27 \%$, para dejar al final la compra de artículos electrónicos y electrodomésticos con un 19.49\%.

El habitante de la ciudad de Quito, en un $60.22 \%$, prefiere realizar sus compras de alimentos en supermercados, si no es posible hacerlo en estos establecimientos, por la razón que fuere, el $45.69 \%$ busca un mercado tradicional de la ciudad y si el tiempo apremia y es poco lo necesitan comprar, el $38.18 \%$ de las personas lo hacen en la tienda del barrio.

Los artículos electrónicos y electrodomésticos se encuentran en el último lugar de preferencia de compra con relación a otras necesidades que tiene el habitante de Quito; sin embargo, son considerados como un hábito general de gasto, puesto que, se los debe adquirir por alguna necesidad impresindible como la culminación de la vida útil del bien o porque realmente es necesario comprarlo para facilitar las actividades del hogar, en especial equipos con nuevas tecnologías que ayudan en tareas que generan desgaste físico.

La investigación revela que el $66.45 \%$ de las personas prefieren comprar electrodomesticos en lugares especializados en estas líneas de productos, simplemente porque ellos tienen las garantías necesarias en cuanto a conocimiento y servicio técnico especializado. En segundo lugar, un 55.27\% optan por comprar en cierto tipo de supermercados, que a más de comestibles, poseen internamente departamentos de ropa, zapatos, cosméticos, jardinería, electrodomesticos, muebles económicos, neumáticos para autos, cafetería, librería y otros departamentos especializados. Existe un atractivo especial por este tipo de supermercados debido a que tienen ofertas de descuento con mucha frecuencia. El internet es una tercera alternativa para el 30.19\% de los habitantes, esto se debe, según la investigación, a que esta alternativa está superditada al conocimiento y confianza que tienen los ususarios en este tipo de transacciones, por un lado, y al buen manejo de la herramienta por otro.

La ropa es el segundo hábito general de compra en las personas que viven en la ciudad y su preferencia en un $63.42 \%$ es por los almacenes de venta que tienen variedad de marcas a la disposición de los clientes, seguido muy de cerca, con el 57.03\%, por los almacenes 
ANÁLISIS DEL PATRÓN DE COMPORTAMIENTO DEL CONSUMIDOR DE QUITO, ECUADOR. especializados en cierto tipo de ropa, como casual para hombre o mujer, ropa para niños, deportiva, para compromisos de etiqueta, bodas, bautizos, fiestas especiales, entre otros. Como una alternativa no muy usual, están las compras por internet con el 39.30\%.

Cuando quieren elegir el lugar de compra, el $63.10 \%$ se fija, en primera instancia, en las promociones destinadas al bien que buscan, en segundo lugar, un $45.85 \%$ buscan facilidades de pago; y por último, para la decisión de ir o no al lugar de compra, el $41.21 \%$ revisa la ubicación y estacionamiento para clientes. Cabe destacar, que la misma importancia que le otorgan a la ubicación y al estacionamiento, le confieren a la gentileza y amabilidad del servidor, asi como la facilidad de encontrar los productos dentro del lugar.

Si se trata de buscar un bien en particular, el $64.22 \%$ lo hacen en lugares que comercializan productos de calidad, al $48.40 \%$ le interesa que los precios del producto sean competitivos y precio sea de promoción. La disponibilidad del producto y la varidad de marcas que comercialice el lugar de compra, es la tercera alternativa para un $47.28 \%$ de los quiteños.

En el momento de decidir la compra, las personas que viven en la ciudad tienen autonomía para hacerlo. Los resultados de la investigación revelan que el $52.40 \%$ de las personas deciden por si mismas, el $50.80 \%$ consulta a la familia y un $43.45 \%$ a su pareja o amigos. Estos resultados, no distantes entre sí, demuestran que la familia y los amigos todavía tienen un gran peso en la toma de decisiones en la sociedad quiteña.

En todos los modelos del comportamiento del consumidor, la familia es un elemento influenciador en la toma de decisiones de compra, forma parte del grupo de referencia y los recuerdos que las personas tienen de las experiencias familiares llegan a ser un referente. El ser humano, obtiene mucha información mientras permanece en el seno familiar, hereda costumbres, buenas prácticas, comportamientos éticos, inclinaciones hacia cierto tipo de productos, como la comida, los lugares turísticos; se nutre de normas, principios y valores; de una u otra manera, lleva una impronta familiar internamente, que en la mayoría de los casos es el elemento decidor.

Un tema muy delicado y sumamente importante es el poder adquisitivo de los habitantes de la ciudad y conocer la forma de realizar el pago de sus bienes. Las personas en un $69.01 \%$ prefieren, para compras hasta 500 dólares, hacerlo en efectivo o cheque personal, la segunda opción de pago con el $46.49 \%$ está la tarjeta de débito; y por último, el 38.18\% prefieren pagar con tarjeta de crédito, en el mejor de los casos sin intereses.

Por su preferencia en la forma de pago de sus bienes, se concluye, que los habitantes de la ciudad de Quito, no tienen ninguna confianza en el sistema financiero. El en focus group previo SALAZAR-LOZADA G. M., FÉLIX-RODRÍGUEZ J. F., MÉNDEZ-OLÁN L. A. 
ANÁLISIS DEL PATRÓN DE COMPORTAMIENTO DEL CONSUMIDOR DE QUITO, ECUADOR.

a la encuesta, se preguntó respecto a la preferencia en la forma de pago y claramente expusieron que las entidades financieras de Ecuador, en una economía dolarizada, cobran un exagerado interés en los créditos que otorgan. Según informe del Produbanco (2017), la tasa de crédito de consumo ordinario, tiene un promedio de 17.30\%-en dólares-.

Con relación a las características generales de un bien, independientemente de cual fuera éste, el 55.60\% de los ciudadanos prefieren la facilidad de uso y la practicidad del mismo, muy cerca en preferencia con el $55.43 \%$, están los usuarios que buscan que el bien se adapte a su estilo de vida, en otras palabras, que calce con su estilo; sin embargo, para el $52.88 \%$, es requisito primordial, la existencia suficiente de información sobre el bien que facilite la decisión de compra.

Existen muchas razones por las que las personas compran un bien, lo interesante de la pregunta es identificar lo que sienten al comprarlo. Con esta óptica, las personas de la ciudad de Quito, en un 69.65\% tienen un motivo principal por lo que adquieren un bien, sienten satisfacción de tenerlo, porque están seguros que el bien complace la necesidad del momento. Esta manera de agradarse uno mismo, dista de las otras formas de sentirse complacido como el ganar prestigio al comprarlo, que para el $42.81 \%$ es fundamental, comprarlo porque está de moda y los demás ya lo tienen, preferencia para el 30.19\%, o porque al adquirirlo sienten seguridad frente a los demás.

Los habitantes de la ciudad, se inclinan en un $61.18 \%$ por aprovechar oportunidades que ofertan los lugares de compra relacionadas a productos de calidad con precios únicos, seguido muy de cerca con el $55.43 \%$ por la compra de productos de su interés a precios convenientes, que normalmente son resultado de ofertas en diferentes épocas del año. Como tercera alternativa de compra, distanciada de las anteriores con 15 puntos porcentuales, se encuentran aquellas decisiones que de una $u$ otra forma pasan a ser obligadas, como aprovechar la compra de un nuevo producto para reemplazar otro que ha dejado de trabajar por caduco, su performance ha decaído notoriamente y amerita el cambio.

Los consumidores son influenciados de diversas maneras utilizando un sin número de herramientas que persuaden a la compra. De todas maneras, existen consumidores que son fieles a una marca o a un establecimiento, quizá por la experiencia positiva que tienen con ellos o porque no han encontrado un sustituto que les convenza.

En los resultados de la última pregunta de la encuesta, relacionada a la Influencia de los anuncios publicitarios en la decisión de compra, es notorio que la comunicación invita a la compra, para el $51.60 \%$ de las personas, ésta debe contener algún incentivo promocional que 
ANÁLISIS DEL PATRÓN DE COMPORTAMIENTO DEL CONSUMIDOR DE QUITO, ECUADOR.

sea conveniente para que se concrete la adquisición. Sin embargo, se puede notar que, si no existen anuncios promocionales del bien de su preferencia, el $46.96 \%$ de las personas que viven en Quito igual lo compran porque lo necesitan. Esto quiere decir, que existe en los moradores de la ciudad un apego a la marca o establecimiento de su preferencia.

\section{Resumen concluyente de la investigación cuantitativa}

La investigación realizada tuvo como objetivo, conocer el comportamiento general del consumidor que vive en la ciudad de Quito. Se seleccionó para el grupo focal -focus grouplos temas más relevantes y de carácter general. Las respuestas ayudaron a elaborar las preguntas de la encuesta; estas preguntas no son específicas, sino generales básicas, la cual apoyaron a descubrir la preferencia del comportamiento del consumidor de Quito frente a sus actividades cotidianas.

La tabla adjunta resume el comportamiento estándar de los habitantes de la ciudad cuando toman una decisión de compra, por supuesto que para comportamientos específicos frente a un determinado bien o servicio habrá que incorporar preguntas relacionadas al mismo, de manera que su aporte incremente el conocimiento de ese tipo de consumidor en particular.

Tabla 3

Resumen primera y segunda preferencia de los quiteños en el momento de la compra

\begin{tabular}{|c|c|c|}
\hline 1 & Prioridad de los gastos al año & Alimentos-vestido \\
\hline 2 & $\begin{array}{l}\text { Lugar de Preferencia de Compras de } \\
\text { Comestibles }\end{array}$ & Supermercados - Mercado tradicional \\
\hline 3 & $\begin{array}{l}\text { Lugar de preferencia de compra de } \\
\text { electrodomésticos }\end{array}$ & Lugares especializados - Supermercados \\
\hline 4 & $\begin{array}{l}\text { Lugar de preferencia de compra de } \\
\text { ropa }\end{array}$ & $\begin{array}{l}\text { Que tengan variedad de marcas - Especializados en } \\
\text { tipo de ropa }\end{array}$ \\
\hline 5 & $\begin{array}{l}\text { Lo que le gusta más del lugar de } \\
\text { compra }\end{array}$ & Las promociones - facilidades de pago \\
\hline 6 & $\begin{array}{l}\text { Lo prioritario en el lugar de compra } \\
\text { respecto al producto }\end{array}$ & $\begin{array}{l}\text { Que tengan productos de calidad - precios } \\
\text { competitivos }\end{array}$ \\
\hline 7 & $\begin{array}{l}\text { La influencia externa en la decisión } \\
\text { de compra }\end{array}$ & Decisión propia - pregunto a mi familia \\
\hline 8 & $\begin{array}{l}\text { La forma de pago que prefiere para } \\
\text { sus compras hasta } \$ 500\end{array}$ & Efectivo o cheque personal - Tarjeta de débito \\
\hline 9 & $\begin{array}{l}\text { Énfasis en las características } \\
\text { generales de cualquier producto }\end{array}$ & $\begin{array}{l}\text { Que sea fácil de usar y práctico - Que vaya con mi } \\
\text { estilo de vida }\end{array}$ \\
\hline 10 & $\begin{array}{l}\text { Razones por la que compra un } \\
\text { producto }\end{array}$ & $\begin{array}{l}\text { Siento satisfacción al suplir mi necesidad - Me siento } \\
\text { identificado con los que compran el mismo producto }\end{array}$ \\
\hline 11 & $\begin{array}{l}\text { Razones para aprovechar las } \\
\text { oportunidades de compra }\end{array}$ & $\begin{array}{l}\text { Si hay productos de calidad con precios únicos - } \\
\text { Precios convenientes }\end{array}$ \\
\hline
\end{tabular}


ANÁLISIS DEL PATRÓN DE COMPORTAMIENTO DEL CONSUMIDOR DE QUITO, ECUADOR.

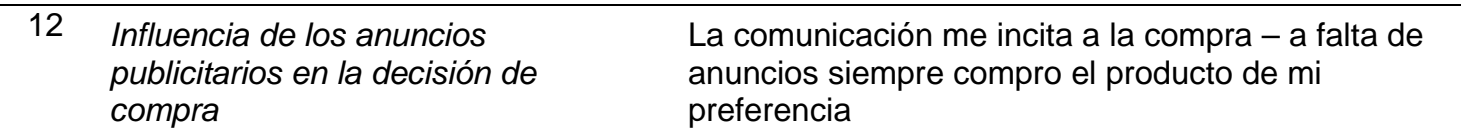

Fuente: Elaboración propia.

Cada tema consultado constó de cinco opciones -alternativas de selección-, el encuestado seleccionó el orden de preferencia de las alternativas. La investigación requería conocer la opción de mayor preferencia. En la siguiente tabla, se puede observar el porcentaje respecto a la muestra total de 626 personas, de la alternativa con mayor preferencia de cada uno de los temas investigados.

Tabla 4

Porcentajes de la preferencia principal del quiteño en el momento de la compra

\begin{tabular}{|c|c|c|c|c|}
\hline & Tema seleccionado & Preferencia Principal & Total & $\%$ \\
\hline 1 & Prioridad de los gastos al año & Alimentos. & 513 & 81.95 \\
\hline 2 & $\begin{array}{l}\text { Lugar de preferencia de compras de } \\
\text { comestibles }\end{array}$ & En Supermercados. & 377 & 60.22 \\
\hline 3 & $\begin{array}{l}\text { Lugar de preferencia de compra de } \\
\text { electrodomésticos }\end{array}$ & $\begin{array}{l}\text { En almacenes especializados en } \\
\text { electrodomésticos. }\end{array}$ & 416 & 66.45 \\
\hline 4 & $\begin{array}{l}\text { Lugar de preferencia de compra de } \\
\text { ropa }\end{array}$ & $\begin{array}{l}\text { En almacenes que tienen } \\
\text { variedad de marcas. }\end{array}$ & 397 & 63.42 \\
\hline 5 & $\begin{array}{l}\text { Lo que le gusta más del lugar de } \\
\text { compra }\end{array}$ & Que tenga promociones. & 395 & 63.10 \\
\hline 6 & $\begin{array}{l}\text { Lo prioritario en el lugar de compra } \\
\text { respecto al producto }\end{array}$ & $\begin{array}{l}\text { Que ofrezca productos de calidad } \\
\text { comprobada. }\end{array}$ & 402 & 64.22 \\
\hline 7 & $\begin{array}{l}\text { La influencia externa en la decisión de } \\
\text { compra }\end{array}$ & $\begin{array}{l}\text { Lo decido yo, sin consultar a } \\
\text { nadie. }\end{array}$ & 328 & 52.40 \\
\hline 8 & $\begin{array}{l}\text { La forma de pago que prefiere para sus } \\
\text { compras hasta } \$ 500\end{array}$ & Efectivo o cheque personal. & 432 & 69.01 \\
\hline 9 & $\begin{array}{l}\text { Énfasis en las características generales } \\
\text { de cualquier producto }\end{array}$ & Que sea práctico y fácil de usar. & 348 & 55.59 \\
\hline 10 & $\begin{array}{l}\text { Razones por la que compra un } \\
\text { producto }\end{array}$ & $\begin{array}{l}\text { Me ayuda a sentirme mejor al } \\
\text { satisfacer mi necesidad. }\end{array}$ & 436 & 69.65 \\
\hline 11 & $\begin{array}{l}\text { Razones para aprovechar las } \\
\text { oportunidades de compra }\end{array}$ & $\begin{array}{c}\text { Su relación calidad-precio es } \\
\text { inmejorable. }\end{array}$ & 383 & 61.18 \\
\hline 12 & $\begin{array}{l}\text { Influencia de los anuncios publicitarios } \\
\text { en la decisión de compra }\end{array}$ & $\begin{array}{c}\text { Si veo un anuncio con una } \\
\text { promoción conveniente, compro } \\
\text { el producto. }\end{array}$ & 323 & 51.60 \\
\hline
\end{tabular}

Fuente: Elaboración propia.

\section{CONCLUSIONES}

Para el habitante de Quito, los alimentos son su primera prioridad, por lo que se entiende que en épocas de recesión económica se privará de muchas adquisiciones que no sean alimenticias. En segundo lugar, los proveedores de alimentos para la ciudad deben centrarse en tener mayor presencia en los supermercados, lugares donde acuden más de la mitad de la población de la ciudad para adquirir alimentos. Para empresas cuyo negocio es la venta de electrodomésticos, es importante que conozcan que el quiteño compra electrodomésticos en SALAZAR-LOZADA G. M., FÉLIX-RODRÍGUEZ J. F., MÉNDEZ-OLÁN L. A. 
ANÁLISIS DEL PATRÓN DE COMPORTAMIENTO DEL CONSUMIDOR DE QUITO, ECUADOR.

tiendas especializadas en el producto, se siente garantizado con el conocimiento y soporte que le brinda el personal calificado del lugar. Por otro lado, al habitante de la ciudad de Quito, se lo incentiva a la compra con promociones, productos de marca y calidad comprobada, así como, almacenes que tengan variedad de marcas a su disposición. El vendedor de los almacenes o negocios, debe ser muy hábil y cerrar la venta en la primera visita aprovechando que el comprador quiteño es quien toma la decisión, no depende de terceros, y lo mejor de todo, es que, su forma de pago hasta transacciones iguales o menores a $\$ 500$ (dólares), es de contado o tarjeta de débito bancario. Los anuncios de las promociones deben ser impactantes en contenido y atracción, si logra impacto con la publicidad el ciudadano comprará.

Un estudio realizado por Ms. Oswaldo Revelo Ron (2017), de la Universidad Católica del Ecuador, Sede Manabí, revela en sus conclusiones que el consumidor ecuatoriano está mejor informado, es más exigente al comprar, le gustan las promociones y un surtido amplio, el carácter impulsivo sigue presente a pesar de que aplaza sus compras, mantiene su objetivo de satisfacción personal, se siente bien gastando, su temor a equivocarse en las compras va creciendo, busca la mejor oferta y no importa si no ha tenido experiencia con el producto en cuestión, cae fácilmente en la novedad, fácilmente gasta su efectivo, el ahorro no está en sus prioridades, la irracionalidad en el comportamiento de compras va en aumento, la publicidad persuasiva le induce fácilmente a la compra.

\section{REFERENCIAS BIBLIOGRÁFICAS}

Ecuadorcifras (01 de marzo de 2017). Tras las cifras de Quito. Recuperado de WW.ecuadorcifras.gob.ec: http://www.ecuadorencifras.gob.ec/tras-las-cifras-de-quito/

Fernández Delgado, R. (2014). Teoría de clase ociosa. Iberian Journal of the History of Economic Thought, 1(2), 2386-5768.

Herrera, F., Ramírez, M. I., Roa, J. M., y Herrera, I. (2004). Tratamiento de las creencias motivacionales en contextos educativos pluriculturales. Revista Iberoamericana de Educación, Sección de Investigación, 37(2). España. Recuperado de: http://www. rieoei.org/investigacion/625Herrera. PDF

INEC. (2017). Reporte de Economía Laboral. Quito: INEC.

Lavoie, M. (2005). La economia postkeynesiana: un antídoto del pensamiento único-Amazon AWS. Recuperado de SALAZAR-LOZADA G. M., FÉLIX-RODRÍGUEZ J. F., MÉNDEZ-OLÁN L. A. 
ANÁLISIS DEL PATRÓN DE COMPORTAMIENTO DEL CONSUMIDOR DE QUITO, ECUADOR. http://ecaths1.s3.amazonaws.com/economia2unrn/1511167762.lavoie_economia\%20p ostkeynesiana\%201.pdf:

López, A. (2013). Las 8 teorías más importantes de la motivación. Recuperado de http://managersmagazine.com/index.php/2013/11/las-8-teorias-mas-importantessobre-la-motivacion/:

Produbanco (09 de 01 de 2017). Tasas pasivas Produbanco. Recuperado de www.produbanco.com.ec: $\quad$ https://www.produbanco.com.ec/media/354921/625formatos-prd-tasas-del-lunes-09-al-martes-31-de-enero-del-2017.pdf

Revelo O. (2017). El comportamiento del consumidor ecuatoriano desde la perspectiva psicológica. Recuperado de http://repositorio.pucesa.edu.ec: http://repositorio.pucesa.edu.ec/bitstream/123456789/2081/1/El\%20Comportamiento\% 20del\%20Consumidor.pdf

San Martín, S. (2008). Prácticas de marketing, ejercicios y supuestos. Madrid: ESIC.

Schiffman, L. y Lazar Kanuk, L. (2010). Comportamiento del consumidor. Estado de Mexico: Pearson. 